\title{
Memorándum para el «poder popular». Migración, precariedad y nuevos movimientos sociales en el post-apartheid en Sudáfrica
}

\author{
CARL-ULRIK SCHIERUP*
}

RESUMEN: Este artículo discute temas de migración, precariedad y movimientos sociales entre los pobres. Aborda la reproducción de la pobreza y el trabajo no-libre en la Sudáfrica post-apartheid como vía para cambiar las alianzas de clase y raza y dar paso a la construcción de una hegemonía política bajo cambiantes condiciones histórico-estructurales. Describe el desarrollo del sistema migratorio sudafricano como itinerario de un régimen de migración laboral dirigido centralizadamente desde el apartheid hacia un régimen neoliberal post-apartheid regido por políticas de «flexplotación». Un controversial enigma de "xenofobia» emerge en torno a los conceptos de nación y ciudadanía en la reconstrucción de la hegemonía en una sociedad excesivamente desigual, donde los movimientos de los pobres crean espacios para una «insurgencia ciudadana» que trasciende las posibilidades de alcanzar una gobernanza neoliberal. El autor se pregunta: żuna reconstituida izquierda sudafricana, con el Tratado Libertario de la coalición anti-apartheid como memorándum para los conflictos en curso, será capaz de propiciar un renacimiento del «poder popular» en una coyuntura donde la migración, la xenofobia y la creciente lealtad de un precariado rebelde se han convertido en estratagemas crecientemente significativos?

PALABRAS ClAVE: Sudáfrica, migración laboral, neoliberalismo, movimientos sociales, extractivismo.

*Director del Instituto de Investigación sobre Migraciones, Etnicidad y Sociedad (REMESO) de la Universidad de Linköping, Suecia.

Traducción de Jorge Miguel Veizaga Rosales. 
ABSTRACT: The article discusses migration, precarity and poor people's movements. It relates a reproduction of poverty and unfree labour in postapartheid South Africa to shifting race-class alliances and the constitution of political hegemony under variable historical-structural conditions. It charts the development of migrancy system from apartheid's centrally managed labour regime to a post-apartheid neoliberal regime driven by policies of «flexploitation». A contentious enigma of "xenophobia» is related to nation and citizenship in the remaking of hegemony in an exceedingly unequal society where poor people's movements carve out spaces of an «insurgent citizenship» beyond the reach of neoliberal governance. The author asks whether a reconstituted South African left, with the anti-apartheid coalition's Freedom Charter as a memorandum for current struggles, will be able to advance a renaissance of "people power» at a junction where migration, xenophobia and winning the loyalty of an unruly precariat have become increasingly crucial stratagems?

KEY WORDS: South Africa, labor migration, neoliberalism, social movements, extractivism. 
1 apartheid ha llegado a usarse como una densa metáfora en los estudios sobre migración y la noción de trabajo no libre o forzado, en contextos geográficos y sociales muy dispares del Norte global y el Sur global, ${ }^{1}$ ha sido usada como un caso arquetípico de hiperexplotación laboral basado en la violencia de clase, exclusión de los derechos ciudadanos, expulsión hacia países de origen sojuzgados y legitimados a través de mitologías de raza, cultura, etnicidad o identidad nacional. Pero el movimiento anti-apartheid y su lucha por una sociedad democrática no racial fue, simultáneamente, una de las luchas emancipatorias anticoloniales más importantes del siglo xx. En este doble sentido, el recuerdo del apartheid ha permanecido como crucial para los estudios migratorios críticos. Es así que los desafíos, rupturas y aún indefinidas trayectorias del desarrollo sudafricano del post-apartheid todavía son esenciales para los estudios sobre migración, poscolonialismo e imperialismo en su apariencia neoliberal de globalización, alegóricamente denominado «apartheid global» (Bond, 2004; cfr. Richmond, 1994).

Una larga lucha contra el régimen sudafricano del apartheid dirigido por el Congreso Nacional Africano (CNA) se dirigía a la victoria al final de los años ochenta. El CNA — apoyado por su alianza tripartita con el Partido Comunista de Sudáfrica (PCSA) y el poderoso Congreso de Sindicatos de Comercio de Sudáfrica (CSCSA) - inició negociaciones con el antiguo enemigo y pilar político del apartheid, el Partido Nacional de los Afrikaners, desde inicios de los

${ }^{1}$ E.g., las condiciones de la «subclase» racializada en Estados Unidos (Massey y Denton, 1993), el régimen de ciudadanía del siglo xxi de la Unión Europea (Balibar, 2004: 121), el «apartheid económico» en la multicultural Canadá (Galabuzi, 2006), el «apartheid social» en Brasil (Hunt, 2007) y Arabia Saudita vista como «El Estado real de apartheid de Medio Oriente» (Greenfield, 2014). 
años noventa. Esto llevó a la eliminación formal del apartheid en 1994 junto con el logro del poder político de un gobierno dirigido por el CNA sobre la base de elecciones democráticas libres. La nueva Sudáfrica nacida de esta Revolución Nacional Democrática, ${ }^{2}$ sería transformada en una Nación Arcoíris inclusiva; una seductora parábola hecha por el Premio Nobel Desmond Tutu (1996) para designar la unidad multicultural del Pueblo arcoíris de Dios en un Estado previamente definido por la profunda grieta del apartheid entre blancos y negros. Era el sueño del «excepcionalismo» de la Sudáfrica no-racial incorporado en la filosofía Ubuntu que habla de la «esencia misma del ser humano» (Tutu, 1999: 31) en términos de los lazos universales de la compasión, el compartir, el cuidado y la generosidad. Abraza el valor africano de la hospitalidad simbolizado por la parábola de Nelson Mandela de «un viajero que va por el país», que «se detendría en un pueblo y no tendría que pedir comida o agua» (Mandela, 2012). Incluye la cualidad del perdón y pacificación que guía la célebre Comisión Sudafricana de Verdad y Reconciliación (CSVR), que se suponía cerraría la era del apartheid, un largo capítulo de opresión de raza-clase y violencia de mutua destrucción. En este sentido, Ubuntu representa esencialmente un tipo sudafricano de discurso humanitario que viene como un "puente de consenso entre la tradición colonial racista reformada de la élite colonialista blanca que va de salida y el nacionalismo africano reformado de los que vienen» (Neocosmos, 2011: 368). ${ }^{3} \mathrm{Sin}$ embargo, para las fuerzas políticas de izquierda en el movimiento contra el apartheid, el establecimiento de la democracia liberal y la igualdad formal de los ciudadanos fue todavía una «revolución inconclusa» (Alexander, 2010). Desde esta perspectiva, Ubuntu significa más que un evangelio humanitario; representa la visión anticapitalista de un contra-movimiento que construiría el poder popular cristalizado como un memorando suplicatorio en el

${ }^{2}$ La idea de la Revolución Nacional Democrática fue formulada inicialmente por el PCSA en 1928 y más tarde fue adoptada como consigna política para la amplia lucha anti-apartheid dirigida por el CNA. El PCSA partió en principio de la idea de Lenin de que la Revolución francesa y, más tarde, las revoluciones burguesas, incluyendo la primera revolución rusa de 1905, eran revoluciones (socialistas) «inconclusas» (Sewell, 2004; Slovo, 1988).

${ }^{3}$ Para una discusión crítica de la CSVR, enfocada en sus limitaciones para establecer una base sólida para la ciudadanía social en la era post-apartheid, véase Barchiesi (1999).

$6 \frac{\text { PRIMER SEMESTRE } 2015}{\text { MIGRACIÓN Y DESARROLLO NÚM. } 24}$ 
Congreso del Pueblo del Freedom Charter en 1995 (ANC, 1955). Se transferiría la riqueza mineral de la República a la «propiedad del pueblo» y se redistribuiría la tierra «a aquellos que la trabajan».

La Nación Arcoíris realmente existente se convertiría en un edificio rocoso diseñado por «arquitectos de la pobreza», sostiene Moeletsi Mbeki (2009). ${ }^{4}$ El origen de las actuales élites de riqueza y poder es, como antes, los recursos sudafricanos subterráneos de metales preciosos y minerales. Antes bien, el cofre de oro de Sudáfrica, su poderoso complejo corporativo mineroindustrial, es — de acuerdo con el agudo análisis de Mbeki-como una trampa tóxica al final del arcoíris. El ilimitado reino del extractivismo depredador ha continuado generando la precariedad del trabajo y de la ciudadanía en un enorme sector de pobres negros dentro de una sociedad todavía profundamente racializada. Además, no está el negro en el arcoíris, insiste Reshoketswe Mapokgole $(2014)^{5}$ en otra crítica exposición. Ella examina la multifacética xenofobia, como «afrofobia» o "negrofobia» que incita a los «nativos» negros pobres en contra de los negros pobres «extranjeros», en una sociedad en que la desigualdad es profunda como el océano y donde el régimen de hiperexplotación migratoria continúa siendo uno de los problemas más controversiales.

En el contexto de estas y otras opiniones más extremas en el debate sobre la actual crisis en la economía, política y sociedad, pretendemos explicar la transición de Sudáfrica y su sistema migratorio que va desde una administración centralizada del trabajo forzado por la burocracia estatal del apartheid hacia un Estado neoliberal de precarización y «flexplotación» (Bourdieu, 1999: 84) determinado por la instrumentalización corporativa de la inseguridad. Seguimos analizando el enigma de la xenofobia y su papel en el mantenimiento y reproducción de la hegemonía en un momento marcado por la «ciudadanía insurgente» determinada por una múltiple «sociedad incivil» a lo largo de «espacios inventados» y más allá del alcance del gobierno neoliberal. Esto se completa con una discusión acerca de las opciones de una reconstituida y contrahegemónica izquierda sudafricana para construir el

${ }^{4}$ El hermano menor de Thabo Mbeki, el segundo presidente post-apartheid de la República estaba asociado con la drástica tendencia de reorientación neoliberal del CNA.

${ }^{5}$ Evocando el trabajo de Paul Gilroy (1987), There Ain't No Black in the Union Black. 
renacimiento del poder popular en una coyuntura marcada por la lucha por los corazones, mentes y cuerpos de un incontrolable grupo precario en ciudadelas multiétnicas y en un mundo laboral informalizado.

\section{Trabajo NO LIBRE COMO UN LEGADO COLONIAL}

El apartheid fue el marco en el que, en 1948, el régimen migratorio y laboral sudafricano se distinguió por «una extrema coerción extra-económica de la mayor parte de la fuerza de trabajo» (Legassick, 1974: 255; cfr. Wolpe, 1972). El trabajo forzado durante el apartheid (1948-1994) puede, como tal, ser visto como una prueba en contra de la doctrina marxista que considera el «trabajo libre» como la quintaesencia del trabajo en el capitalismo: esto es, la libertad del trabajador asalariado respecto a la propiedad de los medios de producción, pero también la libertad básica de vender su fuerza de trabajo a través de la negociación, firma o conclusión de un contrato (Marx, 1976 [1885]). Más ampliamente, reflexionando en las nuevas tendencias de la migración internacional del trabajo en los años ochenta, Miles (1987) y Cohen (1987) sostienen que el trabajo «no libre» refleja más apropiadamente las condiciones reales de una amplia gama de trabajadores bajo el capitalismo, en el pasado o el presente, expuestos a exclusión social, racismo, segmentación discriminatoria del mercado laboral y diferentes maneras de coerción formal e informal y, por tanto, no son capaces de ofrecer libremente su trabajo en el mercado.

Siguiendo con el argumento de Phillips (2013: 172), éste considera al trabajo forzado como una «forma de incorporación adversa» crecientemente común hoy en día, una inclusión excesivamente explotadora de trabajadores pobres y vulnerables en las cadenas globales de producción, concomitante con la reestructuración neoliberal de los mercados de trabajo y de los regímenes migratorios, los cuales en la mayoría de los casos sólo reproducen desigualdad, pobreza y precariedad. Fudge y Strauss (2013) observan que la falta de libertad producida e instituida por el empleo y la inmigración varía en el tiempo y en el espacio, pero ahora es típicamente contingente con

$8 \frac{\text { PRIMER SEMESTRE } 2015}{\text { MIGRACIÓN Y DESARROLLO NÚM. } 24}$ 
las diferentes formas de trabajo intermedio, temporal y flexible que se ve facilitado por el crecimiento de la subcontratación y una gran variedad de agencias de empleo y agentes laborales. Esto implica restricciones en la movilidad de los obreros, que les prohíbe cambiar de empleo y limita su libertad de agencia. En esa misma línea, Benjamin (2013a) insiste en la persistencia del trabajo no libre actualmente en Sudáfrica, aunque el régimen particular que reproduce la falta de libertad ha cambiado drásticamente, esa es una perspectiva que seguimos en la discusión en las siguientes secciones acerca de la transición en la economía política de Sudáfrica y en el cambio en los regímenes administrativos de la fuerza de trabajo.

El apartheid implicó el fortalecimiento de los rasgos diacríticos del capitalismo colonial. Como tal, representó una tercera fase en la estrategia de acumulación capitalista en Sudáfrica, en la desposesión de los negros africanos de su tierra y en la producción y reproducción de un gran sistema de migración laboral, en principio establecido bajo el gobierno colonial británico desde el inicio de la minería de oro a mediados del siglo xix y que se extendió por todo el sur de África. Después de finalizar la guerra sudafricana y de que se estableciera la Unión Sudafricana como un dominio británico en 1910, la élite Afrikaner, que probó tener capacidades para la administración del Estado y para disponer efectivamente de trabajo negro y barato, se convirtió en un aliado estratégico de los blancos anglo-capitalistas que controlaban la industria minera y propició una potencial alianza con la élite negra profesional y de negocios enraizada en el colonialismo británico del siglo XIX (Mbeki, 2009). Las Reservas Nativas —instituidas a través del Código de Tierras de 1913 y basadas en una alianza entre las compañías mineras controladas por ingleses y los terratenientes Afrikaners- significaron un paso más en la alienación colonialista de los negros africanos de sus tierras y fortaleció los fundamentos del régimen de migración laboral. El apartheid efectivamente modificó y además consolidó este proceso histórico de alienación fabricando «extranjeros» a partir de los antiguos «nativos» mediante la lógica del denominado desarrollo separado que está implícita en las empequeñecidas tierras de los nativos llamadas bantustans, quienes estaban impedidos de tener ciudadanía en la «blanca» República de Sudáfrica, y a través de 
medidas reforzadas de administración y seguridad, han sido enjaulados en una permanente condición de migrantes y trabajadores forzados (Benjamin, 2013a).

El control de los granjeros blancos sobre la inmensa mayoría de la tierra agrícola (tomada por los colonizadores durante el siglo XIx) y la carga impositiva discriminatoria sobre los pequeños propietarios negros privó a la población rural negra de la seguridad en la tenencia de la tierra. Esto se combinó con la contratación de trabajo temporal administrado por agencias centralizadas, con la construcción de recintos reglamentados de trabajadores migrantes en las zonas mineras y leyes de movilidad y circulación restrictivas diseñadas para contener los asentamientos urbanos de negros (Frankel, 1979). Las políticas de segregación urbana incluyeron el arrasar barrios racialmente mixtos favoreciendo la creación de villas periurbanas negras. A través de la bantustanización muchos habitantes urbanos perdieron la ciudadanía sudafricana y estuvieron sujetos al régimen laboral establecido. La relocalización de los negros sudafricanos de las villas y las áreas rurales de blancos hacia los bantustans, incluyó políticas de «desalojo forzado» (Henrard, 1995-1996).

El apartheid forzó a millones de negros sudafricanos a migrar entre «tierras nativas» rurales, sobrepobladas y empequeñecidas y recintos mineros, granjas blancas y fábricas en áreas urbano-industriales. Esto fue acompañado por un régimen regional sudafricano más amplio, que proveía trabajo migrante contratado a las minas sudafricanas, a la agricultura comercial y a las industrias. Al igual que la migración dentro del territorio de la posterior República de Sudáfrica, esto se inició a mediados del siglo XIX, cuando la industria minera colonial del diamante y el oro se fundó y continuó durante el siglo xx. En el periodo colonial, las comunidades proveedoras de mano de obra a lo largo de la región de África del Sur fueron controladas por medio de un gobierno indirecto en alianza con las autoridades nativas (e.g., Mamdani, 1996); durante el apartheid, esto continuó a través de la colaboración de Pretoria por medio de los jefes tradicionales en los bantustans y su influencia en la región. Los trabajadores migrantes fueron tomados de todos los estados y territorios fronterizos, ${ }^{6}$ con la mayor parte de los cuales la República logró acuerdos bilaterales de reclutamiento (Wentzel y

${ }^{6}$ Entre otros, los que actualmente son Mozambique, Lesotho, Zimbabwe, Malawi y Swaziland.

$10 \frac{\text { PRIMER SEMESTRE } 2015}{\text { MIGRACIÓN Y DESARROLLO NÚM. } 24}$ 
Tlabela, 2006). En las minas sudafricanas, 40 por ciento de la fuerza laboral estaba constituida por no sudafricanos durante la mayor parte del siglo XX, y cerca del momento de la liberación la proporción era del orden de 60 por ciento (Crush, 2003: 3). La migración a las minas estaba en gran medida regulada formalmente durante el apartheid, mientras que el trabajo indocumentado era más frecuente en la agricultura. Prevalecía la migración circular, que era consistente con el control de los asentamientos de negros en áreas urbanas. La bantustanización de la migración interna implicó innumerables trabajadores no asalariados, principalmente mujeres en las comunidades de origen; una precondición para la reproducción de la fuerza de trabajo migrante hiperexplotada. Pero, mientras se abandonó el control del ingreso de los migrantes de los bantustans en 1986, los trabajadores fronterizos nunca obtuvieron la residencia.

\section{DEL FORDISMO RACIAL A LA PRECARIEDAD NEOLIBERAL}

El apartheid y su sistema integral de trabajo hiperexplotado negro-migranteforzado fue diseñado, principalmente, para atender las necesidades laborales de la industria minera y la agricultura capitalista. Pero pasó a estar en conflicto con las demandas capitalistas en competencia en tanto Sudáfrica se estaba convirtiendo en el Estado en desarrollo más industrializado de África a través de políticas de sustitución de importaciones (e.g., Legassick y Wolpe, 1976). Se trataba de una versión particular de «fordismo periférico» (Lipietz, 1982), un «fordismo racial» (Gelb, 1987), en el cual el dinamismo industrial y la diferenciación económica fueron frenados por el profundo dualismo racial del apartheid, una economía política basada en la extracción de recursos naturales y la estrategia de acumulación predominante heredada del colonialismo. Esta disyuntiva ha sido identificada como un factor importante del declive económico de largo plazo de los años ochenta; además de una situación estructural que ha permanecido con la Sudáfrica democrática del nuevo milenio a pesar de los cambios radicales en el contexto político y en las relaciones de raza y clase (Mbeki, 2009). 
La década que precedió al derrumbe del apartheid, alrededor de 1990, estaba marcada por la recesión económica, sanciones internacionales y crecientes rupturas en la hegemonía blanca. La exclusión de los negros africanos de las profesiones calificadas se volvió cada vez más contraproducente para sectores influyentes del capital. Y no fue menos importante un poderoso movimiento social anti-apartheid - y en particular, la creciente fortaleza de una tendencia de "unidad comunitaria» que se entrometía en los espacios de trabajo, en las villas de negros, constituida enteramente por trabajadores migrantes (Bramble, 2003) - que desarrolló la estrategia de suministrar mano de obra barata en un callejón sin salida. Los beneficios del régimen de acumulación fueron reducidos por las protestas de las masas y huelgas, y para mantenerlos se requirió, cada vez más, recurrir a la policía y al Ejército, lo que implicó inversiones en un aparato de seguridad tan costoso como endeble (e.g., Mbeki, 2009).

La Convención por una Sudáfrica Democrática (Codesa) negoció con el CNA y el Partido Nacional a principios de los años noventa, logró el fin del apartheid y se manifestó a través de las primeras elecciones universales multipartidistas en 1994. Aseguró la democracia política y la ciudadanía para los negros y evitó una prolongada guerra civil, pero aceptó el precio de liquidar valores de igualdad social y políticas de redistribución que eran centrales para sindicatos de comercio y fracciones de izquierda del cNA. También tuvo que ver con el abandono de las demandas de cambios en la propiedad, que durante la lucha contra el apartheid se concibieron como las bases sobre las cuales se lograría el desarrollo económico y social sudafricano justo y sostenible, tales como la nacionalización de las minas y una reforma agraria para el beneficio de los pobres rurales.

En efecto, lo establecido aceleró el desarrollo que ya había despegado durante el apartheid acosado por la crisis en los años ochenta, un caso específico de transformación a partir de un régimen de regulación estatal, Estado desarrollista característico del África postcolonial, hacia un régimen neoliberal (Buhlungu, 2010). El concepto de redistribución para el crecimiento originado en el CSCSA e incrustado en el Programa de Reconstrucción y Desarrollo (PRD) del CNA, originalmente neokeynesiano — con la ayuda de 
instituciones monetarias internacionales - ya en 1996, fue en gran medida cambiado por la máxima de «crecimiento para la redistribución» inscrita en el programa de ajuste estructural neoliberal: la Estrategia de Crecimiento, Empleo y Redistribución (ECER). Ésta implicó el desmantelamiento de las regulaciones de la moneda nacional, la adopción de acuerdos de libre comercio, la reducción de impuestos, la reestructuración de las corporaciones e informalización perjudicial de los derechos laborales y de las organizaciones de obreros, la reducción del Estado de bienestar y la privatización de los servicios públicos. ${ }^{7}$ Entre las consecuencias se cuenta la transferencia masiva de utilidades corporativas fuera de Sudáfrica, ya que se permitió a los capitalistas sudafricanos retirar sus inversiones y riqueza acumulada durante el apartheid, lo que se combinó con una macro — tanto como micro - financiarización tendiente a generar una inercia especulativa y una crisis económica durante los años 2000 (Bond, 2013).

En el proceso, Ronnie Kasrils (2013) —antiguo miembro del comité ejecutivo del CNA - se lamenta en un tono retrospectivo: «la batalla por el alma del Congreso Nacional Africano se ha perdido ante el poder y la influencia de las corporaciones»; un "pacto de Fausto» que otorga a Sudáfrica una economía «tan atada a la fórmula neoliberal global y al fundamentalismo de mercado que ha quedado muy poco espacio para aliviar las terribles condiciones de las masas de[1] pueblo». Más aún, en el trasfondo, el escenario central de lucha por las condiciones post-apartheid fue identificado por Terreblance (2003: 95-96) como una «Codesa II» encubierta; es decir, negociaciones informales entre miembros de la élite política del cNA y la oligarquía blanca con el control de la economía sudafricana. ${ }^{8}$ De ese modo, observa Mbeki (2009: 39-100), la transición refleja el persistente poder e influencia del post-apartheid del Complejo de Minería y Energía (CME) de Sudáfrica, dirigido por la

7 Para un análisis crítico y comprensivo, véase por ejemplo: Maharajh (2011), Hart (2013) y Saul y Bond (2014).

${ }^{8}$ Para tener perspectivas antagónicas y la crítica a Terreblance, véase Dollery (2006) y Tito Mboweni, asesor de Goldman Sachs Internacional, ex gobernador del Banco de la Reserva Sudafricana y presidente de la Compañía Minera Anglo Gold Ashanti, con base en Johannesburgo. Para una discusión de las diversas interpretaciones y perspectivas de las políticas de transición del cna y las causas para la adopción del neoliberalismo, véase Narsiah (2002). 
poderosa corporación angloamericana en una «cuarta fase» (Terreblance, 2003: 95-96) de la búsqueda de una estrategia ventajosa de acumulación en el punto en que el apartheid centralizado por la burocracia de Estado y su amplio aparato de seguridad ha cumplido su papel al proveer trabajo barato a la industria minera bajo la influencia de la sindicalización y la lucha anti-apartheid. Los «oligarcas» del CME han intensificado ahora la búsqueda de una nueva alianza con la clase media negra moderna liberal y urbana de Sudáfrica, cuyos orígenes se remontan al colonialismo británico y que ha sido históricamente una fuerza importante en el interior del CNA. La clave de esta alianza — que depende de la «transición de la élite» consentida (Bond, 2000), implicada en las políticas de reconciliación - consistía en llegar a convertirse en las políticas del llamado empoderamiento económico negro (EEN). Ello implicó la transferencia de acciones corporativas a un número limitado de negros seleccionados denominados individuos anteriormente en desventaja (IADS) —incluyendo prominentes líderes de sindicatos-; se suponía que eventualmente sus efectos se "derramarían» y aliviarían la pobreza entre la mayoría negra. A través de la alianza con una emergente élite política y económica negra, la oligarquía del CME podría retener el control de los enormes recursos naturales de Sudáfrica, mientras que al mismo tiempo, con la ayuda del bloque neoliberal, podrían resguardar su capital contra posibles perturbaciones políticas transfiriendo las oficinas centrales y los beneficios a Londres. Fue un trato ventajoso para el CME, pero con el capital doméstico manufacturero y el trabajo organizado perdiendo espacio. Estaba implícita en el acuerdo una instrumentalización de la globalización en términos de una apertura radical hacia la importación de bienes de consumo industrializados y baratos provenientes principalmente de Asia. Ello reducía los costos del trabajo para el CME. Pero esto significó la competencia internacional destructiva para el sector industrial doméstico que no era del CME, y en consecuencia, un proceso adverso de desindustrialización (Mbeki, 2009), uno de los factores que ha contribuido a la formación de un nuevo e inmenso post-apartheid sudafricano precario atrapado en una red de agencias que generan empleo temporal, contingente e inseguro y la mercantilización de los instrumentos públicos para luchar contra la pobreza.

$14 \frac{\text { PRIMER SEMESTRE } 2015}{\text { MIGRACIÓN Y DESARROLLO NÚM. } 24}$ 
En ésta que es la sociedad más desigual del mundo, cerca de la mitad de la población vive por debajo de la línea nacional de pobreza, la cual, indiscutiblemente, desacredita la agenda neoliberal mercantilista que ha venido a orientar la "guerra contra la pobreza» declarada por los líderes del CNA (Maharaj, Desai y Bond, 2011). El desempleo es alto —entre un 25 por ciento oficialmente y una estimación de 40 por ciento-. Las provisiones compensatorias de bienestar junto con las inversiones en vivienda, servicios municipales y, hasta cierto punto, el creciente acceso a la educación, pueden haber servido para plantear un contrato social entre el CNA y los pobres del país, garantizando la victoria en elecciones parlamentarias consecutivas, pero no alcanzan el imaginado y prolífico «derrame» de la riqueza de la nación hacia los que tienen más desventajas.

La desigualdad promedio interracial de ingreso ha disminuido durante el periodo post-apartheid, pero todavía es amplia. Por otro lado, la desigualdad interracial entre negros sudafricanos se ha elevado. La representación de los negros entre los estratos de profesionales y administrativos ha crecido sustancialmente. Existen cada vez más casos que han ingresado a la élite corporativa a través de - entre otras - la acción afirmativa y el eEN, pero esto ha sido a expensas de la continua explotación de una enorme masa de trabajo barato y precario, particularmente entre mujeres negras y jóvenes, y migrantes internos y fronterizos (Gentle, 2011). La pobreza y el desempleo siguen concentrándose en las antiguas áreas bantustan, pero tendiendo crecientemente hacia asentamientos informales periurbanos, villas negras en condiciones similares a las chabolas o favelas en los países menos favorecidos del globo, y con un elevado flujo de migrantes internos y fronterizos; ya sea que hayan sido despojados de sus casas en las fronteras de la privatización de la vivienda, segregación o proyectos gubernamentales monumentales y de prestigio - los pobres se han concentrado en las denominadas áreas de relocalización temporal (e.g., Ranslem, 2015). La privatización y corporativización de los servicios públicos y la institución de regímenes automatizados de prepago para el caso de las necesidades básicas como el agua y la electricidad en muchas ciudades, tensiona aún más las condiciones de vida de los pobres (Narsiah, 2002). En correspondencia con el libreto 
neoliberal, la responsabilidad de ocuparse de la pobreza de los negros ha sido asignada a los propios negros pobres - entre otras, bajo la forma de «autoayuda» a través de microcréditos- Sin embargo, esto muy difícilmente ha funcionado como instrumento para promover condiciones de vida sustentables, más bien ha servido como un redituable mecanismo de expoliación $y$, en efecto, ha exacerbado las divisiones de raza, clase y género (Bateman, 2012; Hietalahti, 2013).

\section{Vicisitudes de LA fLEXPLOTACIÓN}

El post-apartheid sudafricano puede presumir algunas de las leyes de protección laboral más avanzadas en el mundo. Empero, en correspondencia con la estrategia de acumulación con base en salarios bajos, que en realidad existe y se mantiene, las reglas de las relaciones laborales han sido reajustadas de acuerdo con el discurso neoliberal de la «flexibilidad» (Bezuidenhout y Kenny, 2000). Esto se ha puesto de manifiesto en la legislación que promueve la mercantilización del trabajo, pero nada menos que a través del establecimiento de una situación de flexibilización quo ante bellum a través de la informalización que genera des-sindicalización y precarización del trabajo (Benjamin, 2013a). La eliminación del apartheid como un sistema racial de administración del trabajo legalmente establecido permitió un amplio margen de acción para el reclutamiento mejorado de negros sudafricanos para ocupaciones calificadas para trabajos profesionales de clase media y puestos gerenciales en instituciones del Estado y empresas privadas. Pero simultáneamente se desarrollaron estrategias de triangulación de empleo, orientadas a «externalizar el trabajo» a través de la subcontratación (outsourcing) y, en particular, el traslado de la responsabilidad de reclutamiento, empleo, salario y condiciones laborales desde enormes corporaciones rentables hacia la práctica de una multitud de intermediarios laborales privados. Todo esto ha sido identificado como un determinante de la informalización del empleo (e.g., Altman, 2006) y el crecimiento de nuevas formas de trabajo barato, inestable y precario (Benjamin, 2013a, 2013b; cfr. Bezuidenhout, Godfrey

$16 \frac{\text { PRIMER SEMESTRE } 2015}{\text { MIGRACIÓN Y DESARROLLO NÚM. } 24}$ 
y Theron, 2004). Por tanto, múltiples prácticas corporativas de «informalización desde arriba» (Theron, 2010b) han reemplazado la fuerza extra-económica del apartheid de arriba hacia abajo — basado en la premisa del «desarrollo separado» y la exclusión de los sudafricanos negros de la ciudadanía- como un factor para la producción y reproducción de un ejército de reserva enorme, barato y socialmente inseguro y para la "persistencia de trabajo no libre» (Benjamin, 2013a).

La enorme y precaria masa laboral está compuesta por una muy elevada proporción de negros, en un grado considerable por migrantes y crecientemente femenina. Los espacios que ocupa se convierten en sitios de reproducción de la «informalización desde abajo» (Theron, 2010b) en términos de las estrategias clandestinas de vida desarrolladas por los pobres y que están más allá del alcance de los contextos regulatorios. Un área gris cada vez más amplia existe «donde las fronteras entre la producción formal e informal se hacen borrosas e indistinguibles [...] y donde el empleo difícilmente conduce a la inclusión y la ciudadanía» (Barchiesi, 2010: 68). Se ha registrado que una mayoría de los empleados ha caído en la precaria categoría de trabajadores pobres (Altman, 2006: 11ss), la mayor parte de los cuales «nunca tendrán un hogar decente, ni enviarán a sus hijos a buenas escuelas o disfrutarán de atención a la salud de buena calidad» (Bisseker, 2013). Ellos están ligados a nuevas formas de trabajo forzado, marcado por la represión de la agencia organizada debido a la desregulación, desindicalización, escasez, desánimo, servidumbre de deudas y la disolución etno-racial de solidaridades más amplias. ${ }^{9}$ Es una precarización informal con la cual los sindicatos laborales han perdido contacto (véase Paret, 2013; Hlatshwayo, 2010; Theron, 2010b; cfr. Schierup, 2015).

Los migrantes originarios de los antiguos bantustans estaban a la vanguardia de la lucha contra el apartheid, pero siguen hoy en día entre los más desaventajados de Sudáfrica. A pesar de que el sistema migratorio del apartheid ha dejado de existir formalmente, el legado colonial todavía está

9 Véase Fudge y Strauss (2013), para una discusión general sobre trabajo no libre en el capitalismo contemporáneo. 
más o menos presente (Xulu, 2010: 212). El subdesarrollo y la pobreza en las antiguas áreas bantustan generan las migraciones internas hacia las zonas urbanas donde ellos experimentan el mismo tipo de pobreza que les impulsa a dejar sus comunidades rurales (Xulu, 2010: 212). En tanto trabajadores informales precarios, comparten espacios con la diversidad de los más desaventajados de Sudáfrica, bajo condiciones sociales que a menudo no son menos desgraciadas que bajo el reinado del apartheid. Aquí ellos encuentran una creciente población de trabajadores migrantes irregulares transfronterizos y se hacen compañía con refugiados, a muchos de los cuales se les ha rechazado el asilo. Los migrantes viajan desde regiones vecinas desde las cuales el régimen del apartheid solía reclutar trabajadores, pero muchos también vienen como trabajadores indocumentados de lejanas partes de África, tales como el Congo y Somalia (Tati, 2008).

El centro gravitatorio de la migración transfronteriza se ha movido desde la preponderancia de contratos de trabajo formalmente regulados bajo el apartheid hacia el empleo de trabajadores indocumentados en la década de los años 2000 (Tati, 2008; Gordon, 2010). La adopción de la ortodoxia neoliberal como la orientación política de la Comunidad de Desarrollo de África del Sur (CDAS) y la implementación de programas de ajuste estructural monitoreados internacionalmente a lo largo del África subsahariana ha producido desposesión a través del sobreendeudamiento, apropiación corporativa de los bienes comunes, reducción del sector público, desregulación del mercado laboral, desempleo, depreciación de los salarios y guerras internas de mutua destrucción; también ha producido una creciente confianza en estrategias de sobrevivencia informales basadas en las migraciones fronterizas y el pequeño comercio, con Sudáfrica como destino principal. La constante respuesta sudafricana ha sido contener la migración a través de la reafirmación de la soberanía del Estado, políticas migratorias excluyentes y mayor seguridad (Evans, 2010: 105; Trimikliniotis, Gordon y Zondo, 2008); una condición reforzada por nuevas restricciones legales sobre la migración transfronteriza y la adquisición de la ciudadanía en 2014 (Dube, 2014). Además, el control inconsistente de las fronteras y las prácticas institucionales en el aparato político-administrativo han dado lugar a "fronteras permeables»

$18 \frac{\text { PRIMER SEMESTRE } 2015}{\text { MIGRACIÓN Y DESARROLLO NÚM. } 24}$ 
(Tsianos y Karakayali, 2010) a pesar del discurso oficial restrictivo. La «Fortaleza Sudáfrica» (Crush, 1999; Trimikliniotis, Gordon y Zondo, 2008) — de manera similar a la "Fortaleza Europa» y los regímenes migratorios en las llamadas nuevas «economías de crecimiento» en el África subsahariana (como Nigeria, Ghana y Botswana) — está a provechando la migración irregular y denunciándola al mismo tiempo (Tobias, 2012: 6, citando a Guilfoyle, 2010: 1).

Durante el periodo post-apartheid, los «migrantes ilegales» han sido el blanco favorito del desprecio de los políticos y los medios. Ellos están expuestos al acoso cotidiano de los negros «nativos» que habitan las villas, una condición que se exacerba por el empoderamiento de los oficiales de la policía en el involucramiento con organizaciones de base comunitaria y al educar a la ciudadanía en "asuntos migratorios» junto con la motivación para «'desarraigar' y reportar 'inmigrantes ilegales' a las autoridades estatales» (Neocosmos, 2006: 96-97). Rigurosas redadas policiales en villas periurbanas informales, deportaciones continuas y un régimen restrictivo de ciudadanía junto con fronteras porosas y entradas clandestinas de transportistas privados, intermediarios laborales, la policía y otras agencias públicas (Tshabalala, 2015) se activan a través del nepotismo condicional y la corrupción. Por tanto, la regulación principalmente formal de la migración transfronteriza bajo el apartheid ha sido reemplazada por un régimen informal (Segatti, 2011: 56). De acuerdo con su lógica, «los periodos de 'tolerancia' y 'rigurosidad' alternan convenientemente con periodos de necesidad de trabajo y exceso de trabajo» (Segatti, 2011: 56; cfr. Gordon, 2010); lo cual no es lo mismo que decir que los migrantes tienen que ser necesariamente indocumentados para que puedan ser incorporados en novedosos tipos de trabajo precario informalizado.

Al analizar el caso de los trabajadores migrantes de Zimbabwe contratados en el sector agrícola de Sudáfrica, Theron (2010a) demuestra cómo los intermediarios laborales que intervienen han eliminado la «ideología paternalista» en la que solían encuadrarse las relaciones laborales en las granjas y por tanto han facilitado la «flexibilización» cum precarización del empleo, que se ha observado, en efecto, como la causa de divisiones en el interior de la 
fuerza laboral, dificulta las relaciones laborales entre «locales» $y$ «extranjeros» y genera numerosos incidentes xenofóbicos (cfr., Munakamwe y Jinnah, 2014 [en preparación]). El estudio de Miraftab (2004a) sobre los servicios de recolección de desechos en villas de negros en Ciudad del Cabo presenta otro caso de reestructuración neoliberal enfocado en la privatización e informalización de los servicios públicos municipales sobre la base de trabajo precario. Demuestra la compleja intersección del trabajo en la producción y reproducción y cómo los intereses corporativos son asistidos por estrategias de dominación establecidas a través de la raza o el género. Un creciente grado de informalidad en el sector de hoteles y hospedaje ha reducido los derechos de los trabajadores en su interior y ha hecho más tensa la ya difícil relación entre empresas, trabajadores y sindicatos (MiwORC, 2014), y ha acrecentado la precarización del empleo. Barchiesi (2010) observa que la proliferación del trabajo eventual en el sector manufacturero, en combinación con una creciente estratificación del mercado laboral, socava la solidaridad basada en el lugar de trabajo y limita las posibilidades de organización colectiva. En un estudio del sector de la construcción, Cottle y Rombaldi (2014) registran un creciente nivel de explotación debido a cambios drásticos en el lugar de trabajo, con una decreciente fuerza laboral de empleo regular de tiempo completo y un masivo incremento del trabajo no regulado y no protegido desarrollado por trabajadores precarios no calificados, eventuales, no organizados y predominantemente migrantes vinculados por intermediarios laborales y subcontratistas; un arduo desafío para el movimiento sindical comercial.

Además, la precarización del trabajo y de las condiciones de vida alentada por la estrategia de acumulación post-apartheid del CME llevarán al «Contrato social» del CNA con los trabajadores pobres de Sudáfrica a un punto de quiebre (Cohen, 2013). La masacre en agosto de 2012 de 34 trabajadores mineros por la policía sudafricana, en un intento por romper la huelga contra la compañía minera Lonmin, en los límites de la villa de Marikana en el cinturón de platino Rustenburg, fue el clímax macabro de un prolongado conflicto laboral que involucraba, por un lado, a la compañía Lonmin, el poderoso Sindicato Nacional de Trabajadores Mineros y cuadros dirigentes 
del CNA, y, por otro lado, a mineros luchando por salarios y mejores condiciones laborales a través de la disidencia de la Asociación de Sindicatos de Mineros y Constructores (ASMC), incluyendo numerosos trabajadores migrantes atrapados en los trabajos más riesgosos. El conflicto fue presagiado por un largo proceso de luchas amargas por trabajo y la comunidad y se convirtió en el preludio de más momentos críticos por venir. Se basó en los quiebres en la hegemonía de la alianza política tripartita de Sudáfrica, en la división del trabajo, calificación, ingreso, estatus de identidad y en los intereses y privilegios creados en lealtades verticales y horizontales transversales a la fuerza laboral. Sigue un memorando separado de la CSCSA y la lucha de sus afiliados contra el apartheid que una vez unió a los trabajadores negros entre divisiones étnicas y nacionales, y amplió una visión del trabajo digno entre las villas de negros y los recintos de trabajadores migrantes.

A decir verdad, el jueves sangriento de Marikana, declara Frankel burlonamente (2013: 163), ha sido una «pequeña atrocidad» en el océano menos reportado de la destrucción cotidiana del trabajo, vidas humanas, tierra y dinero en el interior de la industria sudafricana minera. Más allá de los impecables reportes corporativos que muestran el manejo sostenible de la fuerza de trabajo y el responsable desarrollo comunitario de Marikana, se expone el fatal enredo de la reestructuración corporativa y la informalización predatoria del trabajo y las condiciones de vida con la complicidad de los sindicatos, así como del gobierno. La intromisión de intermediarios laborales que contratan trabajadores temporales es un medio frecuente para recortar los estándares laborales (Forrest, 2013). Esto sucede en crecientes sectores del trabajo, incluyendo operaciones mineras esenciales en los pozos (Frankel, 2013: 82ss; Bezuidenhout y Buhlungu, 2011: 251ss). Ello implica una hiperexplotación intensiva del trabajo de un contingente contratado y formado por migrantes laborales precarios provenientes de las mismas y empobrecidas reservas de mano de obra donde el régimen del apartheid reclutaba trabajadores para las minas, dentro de la misma Sudáfrica como en la región más amplia de África del Sur. Expuestos a las prácticas ilícitas de intermediarios inescrupulosos, están forzados a aceptar cualquier empleo, trabajar en áreas riesgosas donde los trabajadores permanentes no irían, aceptar largas 
jornadas que extienden cualquier regulación formaly, según enfatiza Frankel (2013: 99), sin ninguna oportunidad de confiar en la capacidad del departamento de trabajo de monitorear sus propias leyes y reglamentos.

La dependencia respecto a las redes criminales o de traficantes, locales o internacionales, severas prácticas de usureros y cobradores matones y terratenientes de duro puño, les fuerza a asumir una servidumbre por deuda y una condición de facto de trabajador no libre. El rol de los prestamistas de dinero tradicionalmente basado en las comunidades, los mashonishas, ha sido crecientemente asumido por poderosas - pero igualmente inescrupulosasinstituciones microfinancieras administradas por bancos sudafricanos, los cuales han encubierto en las zonas mineras, según reportan Bateman y Sharife (2014), un lucrativo mercado en un «programa deliberado para comprometer a los individuos más vulnerables y explotados del país». Se ha obligado a muchos trabajadores mineros a que asuman deudas impagables, con bancos como el Ubank, entre otros, que tienen al Num como principal accionista. Tal vez éste sea uno de los factores que da lugar a la agonía y rabia que encendió la sangrienta confrontación en Marikana (véase Bond, 2013). Existe un estado de inseguridad y represión entre muchos obreros que residen en la localidad, al igual que entre los migrantes sudafricanos, pero son los migrantes transfronterizos los que se encuentran especialmente en riesgo. Por tanto, el régimen migratorio colonial y de apartheid, basado en la hiperexplotación del trabajo forzado masculino y precario, se reproduce bajo las actuales condiciones informales, junto con mujeres y familias en las zonas rurales pobres que dependen de sus magros ingresos. Las mujeres jóvenes víctimas de tráfico de personas tienen muy pocas oportunidades de empleo y a menudo terminan como prostitutas en las villas miseria que rodean las minas. Estos lugares son los peores barrios en ciudades como Marikana. Estos lugares muestran, en su condición de reflejo espacial, las jerarquías laborales y ocupacionales industriales en las que los trabajadores migrantes externos están en el fondo y con una capa superior de gerencia y control, todavía constituida en su mayoría por blancos, que ha sido mantenida a través de alianzas entre empleadores y sindicatos.

$22 \frac{\text { PRIMER SEMESTRE } 2015}{\text { MIGRACIÓN Y DESARROLLO NÚM. } 24}$ 
Junto a consensos transversalmente horizontales y esfuerzos que unen a trabajadores nacionales y extranjeros, surgen conflictos xenofóbicos y etnonacionalistas que son racionalizados racialmente entre las comunidades mineras y en las propias minas. Ésta es la materia prima para nuevos modos de control, que permiten ver cómo se cierra todo el círculo de las relaciones capital-trabajo. "[El] espacio puede ser usado para conformar, pero también para fragmentar», afirman Bezuidenhout y Sakhela (2011: 254). El apartheid generó conglomerados regulares de migrantes en comunidades mineras segregadas como nodos de control en su sistema de trabajo forzado. Sin embargo, las cosas no se dieron como se esperaba (Bezuidenhout y Buhlungu, 2011: 240), ya que dicho espacio fue ocupado por el movimiento de trabajadores negros como un bastión de la lucha por la liberación. En retrospectiva, 1994 fue el momento en que los líderes sindicales y los activistas comenzaron a posicionarse en el interior del partido gobernante y en las instituciones del Estado y muchos trabajadores negros ascendieron en la escala ocupacional hasta asumir posiciones clave en la jerarquía empresarial. Pero esto también se vio acompañado de nuevos modos de control corporativo. La era del conglomerado regular de trabajadores migrantes se terminó, dando lugar a una estratificación por profesión, ingreso y clase, especializada según los criterios étnico-nacionalistas de los asentamientos de negros en el interior de comunidades mineras racialmente segmentadas como Marikana (Frankel, 2013: 117ss). Bezuidenhout y Buhlungu (2011) concluyen que mientras el control en el régimen del apartheid dependía de la burocracia centralizada y de la contención policíaca, el control corporativo actual está determinado por la segmentación fragmentaria y la diferenciación definidas por el «mercado» e inscrita en la política económica de re-racialización, con la complicidad de la élite política negra y del movimiento obrero. Las políticas de flexibilización e informalización del empleo, el bloqueo represivo de las protestas laborales, combinado con una política migratoria indecisa, enfrentan a los sindicatos a enormes desafíos y difíciles elecciones. Según Hlatschwayo (2010), los migrantes son muy rara vez vistos por los sindicatos como agentes que puedan ser incluidos en una lucha más amplia para cambiar la relación 
de fuerzas en el trabajo y las ciudades. Por tanto, los sindicatos tienen parte de responsabilidad en la persistencia de la xenofobia.

\section{ENIGMA DE LA XENOFOBIA: ¿VÉRTIGO DEL APARTHEID O PRECARIEDAD DEL PRESENTE?}

"Mi acento es ridiculizado y se burlan de mí cuando camino en la calle; muy prejuzgado y discriminado porque mi piel es diferente. Mis hermanos de sangre me llaman "Nigger", "Kaffir", "Nyukunyuku", "Kwerekwere", cucaracha y chocolate». Así resume Ndumiso Mbatha (2013) una extraña síntesis transhistórica y post-apartheid de Kaffir y Kwerekwere; siendo el primero un término de la colonia británica para el nativo negro africano y una denominación común y peyorativa para el sudafricano negro bajo el apartheid, y el segundo el mayor insulto actual que denota al extranjero negro africano con un «acento peculiar», una cultura extraña y con una pigmentación imaginada más oscura que los negros sudafricanos nativos. Esta xenofobia postapartheid implica un oximorón, es una «afrofobia» (Mapokgole, 2014) que estigmatiza y vilipendia al extranjero y tiene su personificación en el migrante ilegal originario del África subsahariana.

Lejos de ser compartidas por todos los sudafricanos, las actitudes xenofóbicas han sido constatadas como igualmente representadas entre «los pobres y los ricos, los empleados y desempleados, hombres y mujeres, blancos y negros, conservadores y radicales» (Crush y Pendleton, 2004: 2). Aún más, es un acoso abierto de negros-contra-negros que sufren los migrantes en las villas pobres de negros que ha venido a constituir el estigma de una «enfermedad» viral de xenofobia en los medios nacionales e internacionales. Alexandra es una de las localidades de negros más empobrecidas de Sudáfrica, vecina de Sandhurst, una de las áreas residenciales más ricas de Johannesburgo y también el principal centro de negocios de Sudáfrica, de intercambio de valores, oro y diamantes. Alexandra continúa siendo el símbolo concreto del mencionado estigma. En mayo de 2008, los ataques a los migrantes

$24 \frac{\text { PRIMER SEMESTRE } 2015}{\text { MIGRACIÓN Y DESARROLLO NÚM. } 24}$ 
en dicha localidad estaban en los titulares de la prensa sudafricana e internacional. Esta chispa se expandió como un incendio por todas las localidades en el país. Dejó más de 60 muertes de migrantes, cientos de heridos, más de 150,000 personas sin hogar y propiedades demolidas o saqueadas; la mayoría de las víctimas eran migrantes transfronterizos, pero también trabajadores migrantes sudafricanos que se supone no pertenecían a la comunidad local.

Aunque brutalmente separados, y a pesar de que parezcan realidades independientes, los opulentos asentamientos como Sandhurst y las villas miseria como Alexandra, actualmente, y no menos que durante el apartheid, se encuentran íntimamente conectados (Mingxitama, 2008: 197). Al igual que en otras ciudades globales del mundo, la riqueza históricamente acumulada de zonas como Sandhurst, Johannesburgo — que durante el apartheid las habitaban solamente blancos y hoy en día son compartidas por un creciente número de negros acaudalados - es producida por el arduo trabajo de un creciente conglomerado laboral precario y multiétnico en localidades muy pobres como Alexandra. Al mismo tiempo, los blancos y los negros ricos de clase media fueron los que «expresaron la mayor sorpresa y disgusto por la violencia que tuvo lugar» (Mapokgole, 2014: 45). Según observa Mapokgole, «en un acto de mala fe, [ellos] se separaron a sí mismos de los residentes de Alexandra que cometieron los violentos actos», los cuales fueron juzgados como «incomprensibles». La autora concluye que ello dejó a la violencia en el vacío «desconectado de la vida que ellos, los ricos, vivían», absolviéndolos, por tanto, de cualquier responsabilidad.

Las diversas masacres en 2008 o pogroms (Neocosmos, 2008) fueron solamente un episodio de la violencia infligida a los cuerpos, refugios y propiedades de los trabajadores migrantes, refugiados y vendedores callejeros extranjeros en las ciudades sudafricanas. Todo ello ha sido una característica de la era post-apartheid desde su inicio y sigue siendo una realidad persistente (Crush, 2000; Neocosmos, 2015). El 2015 fue el peor año desde 2008 en términos de violencia contra los migrantes subsaharianos, según el Foro de la Diáspora Africana (ADF 2015), en la previamente mencionada carta-convocatoria al presidente, concluye que «como sociedad, estamos mucho peor que 
en 2008, ya que la actitud y los discursos xenófobos han penetrado las instituciones del Estado y han afectado tanto la base como la cúspide del Estado». Una nueva ola de violencia comenzó en enero de 2015 con ataques contra los pequeños comerciantes somalíes en Soweto. Continuó con ataques mortales a extranjeros que viven y trabajan en Durban, supuestamente promovidos por maliciosas declaraciones en contra de los extranjeros, realizadas por el rey zulú Goodwill Zwelithini y por Edward Zuma, hijo mayor del presidente sudafricano Jacob Zuma. Finalmente, luego de una larga demora, el 16 de abril de 2015 el propio presidente Zuma declaró a la Asamblea Nacional que condenaba los ataques xenofóbicos y las acciones criminales, enfatizando el hecho de que los sudafricanos en general no son xenófobos. Con una actitud políticamente correcta, convocó al respeto por la vida, la dignidad humana, la cohesión social y a Ubuntu, y urgió a todos los sudafricanos a movilizarse contra el racismo, la xenofobia, la homofobia y el sexismo.

A través de una mezcla de cercanía y distancia, separación y rectitud, preocupación moral y mala fe, discurso malicioso y negación políticamente correcta del mismo, en el más alto nivel de la institucionalidad política, las políticas migratorias brutalizadas y Ubuntu, transmitían el significado de la xenofobia como una estratagema política con ramificaciones mucho más extensas que las del comportamiento «incomprensible» de los más pobres de la sociedad. Se trata de un enigma que sigue siendo un asunto de interpretaciones críticas diversas.

Desde una lectura de Franz Fanon y Steve Biko se puede ver la deplorable situación de xenofobia en la Nación Arcoíris como un «vértigo de apartheid» (Matsinhe, 2011), o como un odio del negro infligido a sí mismo (Mbembe, 2015). La xenofobia se representa aquí como un fantasma del pasado "que mancha a la nación con una excesiva negrura» (Matsinhe, 2011: 133). Se ha visto como un antecedente de las prácticas burocráticas post-apartheid bajo la forma de la identificación por catalogación, marginando y separando poblaciones, teniendo a los no nacionales como el equivalente funcional de los negros sudafricanos bajo el viejo régimen (Misago,

$26 \frac{\text { PRIMER SEMESTRE } 2015}{\text { MIGRACIÓN Y DESARROLLO NÚM. } 24}$ 
Landau y Monson, 2009). Esto devela la necesidad de «exorcizar los demonios internos» (Landau, 2011).

Otra explicación que se encuentra a menudo busca exponer por qué «Ocurrió» la xenofobia (e.g., Bond, Ngwane y Amisi, 2010: 11) orientándose hacia la violencia de pobres-contra-pobres como una consecuencia de persistentes desigualdades de raza y clase. Gordon (2010) desarrolla un marco explicativo más amplio, de leyes, política económica y luchas respecto a los privilegios de la ciudadanía. El autor argumenta que la «división entre ciudadano y extranjero» está estipulada a través de la discriminación jurídica que hace eco en el sentido común del discurso político, los medios y la tradición popular compartida entre la comunidad. Más aún, todo eso no funciona simplemente para destacar la «diferencia» genética o cultural, sino para «crear la vulnerabilidad particularmente intensa que deja expuestos a los migrantes a formas de violencia y explotación» (Gordon, 2010: 7). Para que realice esta función, «tales migrantes deben estar exentos de las normas constitucionales que fueron diseñadas para proteger las libertades individuales». Esta es una condición forjada a través de la representación discursiva del «"Estado de excepción", visto como el derecho del Estado a la autodefensa».

La «xenofobia» realmente existente y producida por el Estado, articulada por el acoso diario a los migrantes en localidades pobres y crecientemente en guetos del centro de las ciudades de Pretoria, Johannesburgo y Durban, emerge aquí como una precondición y pena corporal para la ingeniería de un régimen jurídico-político de una multitud «xeno-racial» de «nuevos negros» (Aludiendo a Sivanandan, 2001) que son trabajadores migrantes pobres, tan «terrible como se pueda entender» (Mbembe, 2015). Ellos llegan a ser las principales víctimas, en tanto las luchas por la obtención de ciudadanía, derechos sociales y económicos, beneficios y acceso a los servicios públicos, prometidos por la transición a la democracia se hacen cada vez más ásperas en las villas de negros en las que se exponen a una perjudicial flexibilización del trabajo, desempleo y subempleo, la informalización de las condiciones de vida, la corporativización de los servicios públicos y el encierro en la trampa de deuda de una industria de microcrédito 
que además destruye «escasas reservas de confianza comunitaria inter e intra-étnica, mutualidad, reciprocidad y solidaridad» (Bateman, 2014: 19), al punto de dar rienda suelta a la violencia mutuamente destructiva. Por tanto, el sistema racista del apartheid, de «maximización del trabajo barato con poca carga financiera para el Estado» (Desai y Walsh, 2010: 12) ha sido suplantado por un

contingente de trabajo barato sin derechos al interior de un contingente de trabajo barato formado por negros sudafricanos [...] miembros de una sociedad sin el apoyo el Estado [...] controlados por la violencia tanto del Estado como de otros sudafricanos pobres que se ven a sí mismos como portadores (y posibles beneficiarios) de ciertos derechos y concesiones.

Una multitud de actores marginados, que tienen en común la falta de protección, vulnerabilidad extrema y dependencia en acuerdos institucionales difusos puede parecer — en efecto- un espacio fértil para la proliferación de una sociedad sin restricciones del tipo: todos-contra-todos, con la "psicología de la violencia» operando «sobre la base del eslabón más débil» (Mingxitama, 2008: 196). No obstante, las políticas discriminatorias que procuran la precariedad para la flexplotación, al igual que el estigma postcolonial, la desigualdad y la exclusión racializada, pueden «conducir a cualquier tipo de reacciones diferentes desde la autoinmolación hasta la lucha de clases» (Neocosmos, 2015); y, al final de cuentas, a pesar del diseño específico del apartheid estatal de divisiones etnorraciales los trabajadores migrantes de la gran región de África del Sur, junto con los «nativos-extranjeros» desnacionalizados de los bantustans sudafricanos (Neocosmos, 2006), fueron una parte esencial de la lucha común contra el apartheid.

Este es precisamente el acertijo en el centro del rompecabezas xenofóbico para abordar un análisis más complejo, argumenta Neocosmos (2006). Desde esta perspectiva, la xenofobia aparece como un extraño producto de la realmente existente Revolución Nacional Democrática, lo que exige implícitamente la reconstrucción de una peculiar discrepancia entre, por un 
lado, una práctica y un discurso panafricano inclusivo y altamente movilizador, desarrollado por una ciudadanía activista durante el decisivo periodo de la lucha anti-apartheid en los años ochenta - lo que incluye a los migrantes de los bantustans y de toda la vasta región de África del Sur- junto a una amplia comunidad apoyada en el sindicalismo comercial como vanguardia política; por el otro lado, una estrecha concepción de ciudadanía xenofóbica y pacifista que marca una ideología estatal post-apartheid de construcción nacional sobre la base de una nueva y encubierta hegemonía de raza-clase negra y blanca. Aquí, en nombre de los derechos humanos y ajustando cuentas con las injusticias históricas, el fantasma del pasado está representado no como "xenofobia», pero sí como migración, igualada per se con el maligno sistema de trabajo forzado, que debe ser justamente expulsado al basurero de la historia. Esto se enmarca en declaraciones políticas afrofóbicas y una legislación que exacerba la brutalidad policíaca, la corrupción y la complicidad de la comunidad en la internalización del control de la migración y de la expulsión de los «migrantes ilegales». Lo cual refleja las profundas estructuras del populismo chauvinista.

\section{¿A DÓNDE SE DIRIGE LA REVOLUCIÓN INCONCLUSA?}

Una interminable violencia xenofóbica ha planteado las contradicciones de una crisis política y social cada vez más profunda de manera abierta y ha impulsado a la crítica sobre las discrepancias entre el sueño y la realidad en un país «encerrado en la espiral descendente de la trampa de la pobreza y una espiral ascendente de la trampa de la desigualdad» (Naudé, 2013). El abrazo del neoliberalismo ha "robado el sueño sudafricano», se lamenta Satgar (2011). Las pasadas dos décadas de integración de la República a los circuitos globales de acumulación han dado fin a la credibilidad del discurso positivo post-apartheid del «excepcionalismo sudafricano» y convirtió a Sudáfrica en uno de los muchos «laboratorios» regionales y nacionales del neoliberalismo africano discriminatorio (Satgar, 2012). Alexander (2010) se 
lamenta de encontrar en "Sudáfrica: una revolución inconclusa», las temibles consecuencias del rumbo marcado por el acuerdo político neoliberal post-apartheid;

un horrible mundo de [...] barbarie capitalista con sus devastadores resultados de elevado y creciente desempleo, creciente desigualdad social, terrible violencia criminal, conflictos racistas y xenofóbicos de mutua destrucción [...] muy lejos de la casi utópica euforia revolucionaria con la cual muchos sudafricanos emitieron tan orgullosamente sus votos, ignorantes de lo que se acordó en los diabólicos detalles del proceso de negociación [...] [en] 1994 (Alexander, 2010).

Empero, a pesar de la caprichosa forma de la «maldición de los recursos naturales» (Naudé, 2013) y un sector manufacturero cada vez menos competitivo, Sudáfrica sigue siendo «excepcional» de alguna manera en el contexto más amplio regional y africano; esto es, en su rol como una hegemonía subimperial heredada del Estado apartheid, aunque revestido con nuevas formas. Sudáfrica sigue siendo la economía más grande de África y se perfila como una fuerza regional en la «Nueva Carrera para el África» (UK, 2014) con demandas para un cambio de régimen que tiene diversos nexos con la política de inversiones y préstamos (e.g., Ndletyana, 2011). Es la «Fortaleza Sudafricana» que ha reformado un sistema migratorio y de trabajo forzado con orígenes coloniales por toda la región de África del Sur y el África subsahariana. Es al mismo tiempo una sociedad en que los imaginarios xenofóbicos representan la concreción de una ideología de excepcionalismo que presenta a los «sudafricanos como superiores a los del resto del continente [... ] [y] a los estimados seres humanos que exhiben diferencias de las normas establecidas como extranjeros en la comunidad y por tanto enemigos de la nación, que pueden, por lo tanto, convertirse en legítimos blancos de la violencia» (Neocosmos, 2015).

El crisol de Marikana — «la democracia de Sharpeville» (Frankel, 2013: 4ss) - representa un punto de quiebre. Planteó que la crisis política y social sudafricana requiere ser comprendida con el trasfondo de la crisis de la globalización neoliberal en general y en particular con la estrategia de acumulación

$30 \frac{\text { PRIMER SEMESTRE } 2015}{\text { MIGRACIÓN Y DESARROLLO NÚM. } 24}$ 
post-apartheid con su dependencia unilateral en el extractivismo predatorio forjado por la oligarquía CME sudafricana. Podríamos ver desde esta perspectiva que el jueves sangriento de Marikana es el presagio de una nueva fase crecientemente violenta en la búsqueda de una estrategia de acumulación viable que, hasta ahora, no tiene ningún consenso a la vista. De manera más optimista, en un tono como el de Polanyi (1957 [1944]), tal vez podríamos ver una leve crisis en la estrategia de acumulación neoliberal que viene junto con un «contra-movimiento» diverso y popular que cuestiona la «acumulación por desposesión» (Harvey, 2005) que ha derrumbado el sueño y la lucha por justicia social, bienestar y dignidad que los sudafricanos pobres depositaron en la Revolución Nacional Democrática.

Para el movimiento de los trabajadores — destruido con la complicidad de sus líderes en las estrategias de gestión neoliberal y separado de las comunidades y trabajadores migrantes en el fondo de la sociedad (Theron, 2010b) - la trayectoria neoliberal significó "una paradoja de la victoria» (Buhlungu, 2010). En tanto los sindicatos laborales son debilitados a través de la reestructuración corporativa y la transición de la élite, y en tanto la promesa del inicio del trabajo digno y la ciudadanía inclusiva es ensombrecida por la pobreza, el trabajo precario y la servidumbre por deudas, grandes sectores de trabajadores sindicalizados y muchos trabajadores migrantes se estancan en los trabajos más difíciles a cambio de salarios insignificantes. Esto ha sido radicalizado por el crisol de Marikana. Una tormenta subsecuente de inestabilidad laboral en todo el país ha producido una profunda división en el movimiento laboral. El resultado de aquello todavía es incierto.

Sin embargo, un sector informal precario permanece en los márgenes del movimiento laboral que ha liquidado su celebrado «sindicalismo comunitario", alguna vez arraigado entre las localidades pobres. En su lugar, ha surgido una "ciudadanía insurgente» (Miraftab, 2009) que tiene a «los pobres» como un nuevo sujeto político (Desai, 2002). Se expresa durante los años 2000 como una resistencia micropolítica diaria a las privatizaciones, desahucios forzados y la mercantilización de las necesidades básicas como la electricidad o el agua (Ngwane, 2011; Gentle, 2011; Hart, 2013). En 
contraste con los «espacios invitados» para la deliberación de accionistas con la sociedad civil, empresas y Estado, los pobres crean sus propios «espacios inventados» como lugares para la protesta y la acción comunitaria no solicitada (Miraftab, 2009). Este movimiento informal de los pobres sudafricanos, al margen de cualquier derecho sustancial y más allá del control del Estado, también ha sido caracterizado, siguiendo a Chatterjee (2002) como una «sociedad in-civil» activista (Neocosmos, 2011). Es un movimiento de los desposeídos, seguido por un creciente movimiento de estudiantes negros militantes que cuestionan la supuesta subordinación de la élite política al legado colonial de la nación. Se puede entender como separada de una apreciada sociedad civil de organizaciones de la sociedad civil (OSC) y organizaciones no gubernamentales (ONG) orientadas a lograr la despolitización de la agenda de derechos humanos y ligada mediante su incorporación en el gobierno neoliberal.

Sin importar que suscribamos o no la controversial definición de precariado de Guy Standing (2011) como una nueva, particular y peligrosa clase, podríamos estar de acuerdo con la premisa de que es - en efecto- "peligrosa». Pero es "peligrosa» en el sentido de que es una sociedad in-civil, es decir, flotante, pero un sujeto genuinamente "político» situado en los márgenes de la informalidad. Es indisciplinado y no confiable y está más allá del alcance de la mentalidad gubernamental que coopta, disciplina y despolitiza a la sociedad civil autorizada. Por tanto, la creativa «informalización desde abajo» de los pobres contiene en ella más que un ajuste flexible y afirmativo a la «informalización desde arriba» corporativa. Es una semilla que puede desarrollarse como rebelión. Una transmutación de facilitador de la «flexplotación» a una resistencia guerrillera a la «tiranía de los mercados» (Bourdieu, 1999). De tal manera concebida, la proliferación de la sociedad in-civil sudafricana incluye una multitud de nuevos movimientos sociales y comunitarios, entre ellos el Movimiento de los Desempleados, el Movimiento de los Sin Tierra inspirados por los movimientos latinoamericanos de pobres. La cita del presidente del Movimiento de los Desempleados ilustra esfuerzos vinculados con el mundo que podrían arraigarse en un espacio 
inventado, más allá de actos espectaculares de protesta, pero también indica una visión política más amplia:

En vez de apoyarse en la vieja retórica de lemas vacíos, estamos experimentando con nuevas ideas de solidaridad. La gente está hambrienta, por eso estamos alentando a los hogares a que construyan jardines. Tenemos iniciativas tales como la cocina del pueblo, incluyendo panaderías. Algunas de las luchas más largas y fuertes en el país han invertido mucho en proyectos de construcción tales como guarderías. Tenemos que aprender de esto. Iniciativas como éstas ayudan a construir nuestras comunidades y ayudan a crear plataforma, nuestra tarea secundaria de infundir conciencia social y política y construir un movimiento masivo (Kota, 2014).

La multitud rebelde sudafricana del presente cuenta también con organizaciones y movimientos críticos originados en las comunidades de la diáspora africana, que levantan sus voces en defensa de condiciones de vida simples y contra la xenofobia (e.g., ADF, 2015; Willén, 2015). Sin embargo, estas voces son marginadas, argumentan Desai y Walsh (2010), a favor de recomendaciones civiles al Estado a través de reportes inmaculados financiados por organizaciones internacionales de derechos humanos; el mismo Estado es en muchas formas cómplice y oficialmente la denuncia juega con fuego tanto al alentar como al explotar la violencia xenofóbica. En tanto las profundas divisiones sociales en Sudáfrica se dirigen hacia un punto crítico y en tanto se ha abierto un creciente mar de desconfianza entre los líderes del CNA y el electorado, la xenofobia resulta una estratagema crucial para un bloque de poder aún hegemónico en la actual competencia política.

Salpicar un proyecto de transformación económica mal gestionado con populismo limitado podría reflejar el propio y preclaro interés de la élite del CNA de retener la legitimidad frente al pueblo, señalando las fronteras de pertenencia (Peberdy, 2001). Para Hart (2013), se trata de una estrategia para gobernar — en referencia a la teoría de la hegemonía de Gramsci (1971) y la original perspectiva de Laclau sobre el populismo (1977) — para contener la crisis social y para cooptar, redirigir, apaciguar y explotar políticamente al 
diverso y todavía difuso precariado en la Sudáfrica actual. Su trabajo explica la articulación específica en Sudáfrica del problema general de la transición de la élite en el postcolonialismo. Este problema fue planteado por Franz Fanon (1967) y consideraba que «los nacionalismos postcoloniales, son al mismo tiempo, crucialmente importantes y tremendamente peligrosos» $\mathrm{y}$ remarcaba lo difícil del trabajo implicado en la «desnaturalización del nacionalismo» (Hart, 2013: 24). Con respecto a la Sudáfrica del post-apartheid, la agenda política del CNA se considera como, paradójicamente, portadora de una reproducción peligrosa de las estructuras centrales del apartheid, a través de la re-circulación mitológica del imaginario de la Revolución Nacional Democrática por medio de la coalición hegemónica de raza y clase (Hart, 2013), re-circulación en el interior del cambiante comportamiento del organicismo integral que aprecia lo endógeno (cfr. Willén, 2015).

Una limitación a la creatividad política de los espacios inventados de los pobres puede ser, y de hecho está, instrumentalizada por medio de la política de «el azote hace a los perros». Los activistas que se atreven a ir más allá de los estrechos espacios de invitación (Miraftab, 2004b) para la deliberación democrática, inventan sus propios espacios no solicitados para realizar actos de protesta contra los desahucios, privatizaciones, corrupción, nepotismo, desempleo y condiciones de vida y trabajo en deterioro. Estos activistas a menudo se encuentran con balas de goma, gases pimienta y acusaciones criminales (e.g., UPM, 2013). Sin embargo, el uso excesivo de la fuerza no es una señal de fortaleza de la hegemonía, sino de su inminente crisis. La política de bienestar social es un dispositivo adecuado para el consenso que genera hegemonía y se pueden mencionar diversos ejemplos a lo largo de la historia, tales como la Ley de Pobres inglesa, la contención de Bismark del incipiente movimiento laboral alemán, los conglomerados de trabajadores de élite en el socialismo realmente existente, el New Deal y el Estado de bienestar, kemalismo, peronismo y las políticas de la Nación Arcoíris de apoyo social y de provisión de servicios para los pobres. Empero, su poder de integración ha sido crecientemente reducido por la privatización, mercantilización y financiarización. En esta coyuntura, el desarrollo de ideas políticas neoconservadoras para monitorear la formación

$34 \frac{\text { PRIMER SEMESTRE } 2015}{\text { MIGRACIÓN Y DESARROLLO NÚM. } 24}$ 
de identidad entre los más desaventajados, ha probado ser el esquema favorito para re-estilizar la hegemonía bajo condiciones neoliberales ( $O$ 'Brien y Penna, 1998), lo cual parece tener un creciente impacto día a día.

$\mathrm{Al}$ igual que en otros lugares, y no menos que en los cambiantes escenarios políticos de una «Europa Completa» (Holmes, 2000), la política de nacionalismo endógeno se ha convertido en la clave para el gobierno de los pobres en Sudáfrica (Hart, 2013; Willén, 2015). Esta política expresa o configura la re-tradicionalización actual de Sudáfrica, lo que conlleva entre otras cosas un patriarcado estilizado posmodernamente, con los viriles modales del propio presidente Zuma como un modelo para las familias negras rotas entre los pobres de la nación (Hart, 2013). También pareciera que se replicara la intención del apartheid de aliarse con los «jefes tradicionales», manipulados para contrarrestar las rebeliones urbanas de aquel entonces, así como al cada vez más fuerte sindicalismo comunitario transétnico. En esta perspectiva, una política tradicionalista de endogenismo puede volver bajo la forma de una fragmentación etnonacional balcanizante de la «democracia no racial» ${ }^{10}$ liberal de la Nación Arcoíris, de la cual, la confrontación entre nativos y extranjeros de las naciones fronterizas, será solamente una faceta.

No obstante, el CNA no es el único que compite por los cuerpos, mentes y almas del incontrolable precariato sudafricano. En un contexto de agravada crisis económica, social y política, le hace compañía a los nuevos y crecientemente articulados proyectos contra-hegemónicos de la izquierda (Munusamy, 2015). El poder financiero alternativo que emerge entre los BRICS puede considerarse como una ventaja. Constituye una opción potencialmente más independiente y se vincula con la oligarquía interna, así como con los INDs tradicionales bajo la supervisión del Norte global, lo que aparenta ser una trayectoria cada vez más creíble.

Uno de los resultados más controversiales de la izquierda es el carismático ex líder de la liga juvenil del CNA, Julios Malena, quien fundó el partido Luchadores por la Libertad Económica (LLE), después de haber sido excluido del CNA al haber acusado públicamente a sus líderes de complicidad

${ }^{10}$ Como, por ejemplo, lo previsto por Marais (2001 [1998]). 
en el caso Marikana. A favor de Malena se puede mencionar que es uno de los pocos líderes políticos y miembros del parlamento que se atrevió a visitar las localidades de negros relacionadas con la explosión xenofóbica del 2015. Al hablar en Alexandra, pidió a los nativos pobres, residentes en hacinamiento, que dejaran de ser violentos con sus hermanas y hermanos negros que viajaron para quedarse en Sudáfrica, desde más allá de las fronteras, que más bien se unieran con ellos para luchar contra la corrupta coalición gobernante, identificada como el verdadero progenitor de una miseria compartida. Sin embargo, lo que resulta más provocador es que el LLE ha vuelto a incluir en la agenda política de la nación cuestiones del Freedom Charter hace tiempo olvidadas, incluyendo, en particular, la nacionalización de las minas y una reforma agraria radical. Sin sorpresa alguna, esto ha llevado a los oráculos del capital corporativo a pronosticar una devastadora tormenta sobre el arcoíris. Esto hace recordar que los líderes africanos con ambiciones antiimperialistas viven peligrosamente. El movimiento no resuena en los pobres y para muchos el Lle se distingue como un germen de esperanza, una potencial réplica sudafricana de los movimientos de izquierda populista de Venezuela, Bolivia, Grecia y España. Pero se mantiene en pie a pesar de la dura crítica desde todo el espectro político. Malena es calificado por diversos oponentes políticos como "populista» y demagógico, un «Hitler en proceso» y sus partidarios uniformados (con sobretodos rojos) como una réplica de las tropas de asalto $S A$.

Otro movimiento sudafricano contra-hegemónico es una amplia coalición de fuerzas inclinadas a la izquierda que se reúnen alrededor del recientemente fundado Frente Unido (Fu) con su columna vertebral en los sindicatos laborales que dejaron de estar alineados con la CSCSA y con el CNA después de Marikana. El fu, que al igual que el LLe, ha cambiado su dirección en 180 grados hacia el pasado, podría convocar más convincentemente los recuerdos que subsisten de las pasadas luchas por el «poder popular». Con la atención enfocada en las promesas traicionadas del Freedom Charter, ejerce presión desde la izquierda sobre el cNA y su coalición tripartita para hacer algo respecto a la trayectoria neoliberal que se dirige hacia un callejón sin salida. El movimiento de los pobres, la migración y la xenofobia son sus tres 
temas principales; desde su perspectiva, el dar significado a una conciencia que fortalece e impulsa una izquierda sudafricana revitalizada, requerirá apoyarse en la renovada legitimidad de los pobres informales de la nación y mantener la tendencia hacia un discurso orgánico excluyente de la nación. Esto tendría que ver con la necesidad de los sindicatos laborales de recuperar su propio patrimonio histórico de «sindicalismo comunitario». Por tanto, retomando a Kota Ayanda en nuestro epígrafe: para ingresar en «un terreno de urgencia como opuesto a la política del Mesías», a través de escuchar a, aprender de y recuperar alianzas con movimientos de precarización y despojo de «nativos» $y$ «extranjeros», para conectar el problema de clase y precarización con las cuestiones de migración y xenofobia.

El escenario está listo, pero no hay puntajes pre-ordenados. Brecha dijo en otro tiempo y lugar de la crisis: «Las revoluciones tienen lugar en callejones sin salida. El futuro pertenece a aquellos que reconocen lo posible, antes de que se vuelva obvio» (Brecht, 1967: 387f, vol. V; traducción del alemán realizada por el autor).

\section{Bibliografía}

ADF (2015), «Open Letter to President Jacob Zuma», Foro sobre la Diáspora Africana, en http://adf.org.za/?q =node/57

Alexander, Neville (2010), «South Africa: An Unfinished Revolution?» The Fourth Strini Moodley Annual Memorial Lecture, University of KwaZulu-Natal, 13 de mayo, en https://www.marxists.org/archive/alexander/2010-unfinishedrevolution.pdf

Altman, Miriam (2006), "Low Wage Work in South Africa», documento presentado en IZA/World Bank Conference on Employment \& Development IZA, Berlín, pp. 25-27 de mayo, en http://www.iza.org/conference_files/worldb2006/ altman.pdf

ANC (1955), «The Freedom Charter», African National Congress, 26 de junio de 1955, en http://www.anc.org.za/show.php?.id=72

Balibar, Étienne (2004), We, the People of Europe? Reflections on Transnational Citizenship, Princeton y Oxford, Princeton University Press. 
BARCHIESI, Franco (1999), «Socio-Economic Exploitation, Meaning Contestation and the TRC: Problematic Foundations for a Discourse of Social Citizenship in PostApartheid South Africa», Paper presented at the International Conference on The TRC: Commissioning the Past, Johannesburgo, University of the Witwatersrand, junio de 1999, pp.11-14, en http://works.bepress.com/cgi/viewcontent. cgi article $=1015 \&$ context $=$ franco_barchiesi

(2010), «Informality and Casualization as Challenges to South Africa's Industrial Unionism: Manufacturing Workers in the East Rand/Ekurhuleni Region in the 1990s», African Studies Quarterly, vol. 11, núm. 2-3.

Bateman, Milford (2012), "The Rise and Fall of Microcredit in Post-apartheid South Africa», Le Monde Diplomatique, noviembre, en http://mondediplo.com/blogs/ the-rise-and-fall-of-microcredit-in-post

(2014), «South Africa's Post-apartheid Microcredit-Driven Calamity: Comparing 'Developmental' to 'Anti-developmental' Local Financial Models», Working Paper, Vienna, Austrian Foundation for Development Research (ÖFSE), 47.

Bateman, Milford y Sharife Khadija (2014), "The Destructive Role of Microcredit in Post-Apartheid South Africa», en Milford Bateman y Kate Maclean (eds.), Seduced and Betrayed: Exposing the Contemporary Microfinance Phenomenon, Santa Fe, SAR Press.

Benjamin, Paul (2013a), «The Persistence of Unfree Labour: The Rise of Temporary Employment Agencies in South Africa and Namibia», en Judy Fudge y Kendra Strauss (eds.), Temporary Work, Agencies, and Unfree Labour: Insecurity in the New World of Work, Londres y Nueva York, Routledge.

(2013b), "Law and Practice of Private Employment Agency Work in South Africa», Working Paper, Ginebra, ILO, en http://www.ilo.org/sector/Resources/ publications/WCMS_231442/lang--en/index.htm

Bezuidenhout, Andries y Bridget Kenny (2000), «The Language of Flexibility and the Flexibility of Language: Post-Apartheid South African Labour Market Debates», swor, en http://www.swopinstitute.org.za/node/24

Bezuidenhout, Andries y Sakhela Buhlungu (2011), «From Compounded to Fragmented Labour: Mineworkers and the Demise of Compounds in South Africa", Antipode, vol. 43, núm. 2.

Bezuidenhout, Andries, Shane Godfrey y Jan Theron (2004), «Non-standard Employment and its Policy Implications Precariat», Johannesburg and Cape Town: Sociology of Work Unit, University of the Witwatersrand y Labour and Enterprise 
Project, University of Cape Town, http://www.swopinstitute.org.za/files/ bezuidenhout_et_al_non-standard_employment.pdf

Bisserer, Claire (2013), «Working, but Still Poor», Financial Mail, 1o. de noviembre, en http://www.financialmail.co.za/economy/2012/10/04/working-but-still-poor Bond, Patrick (2000), Elite Transition: From Apartheid to Neoliberalism in South Africa, Londres, Pluto Press.

(2004), South Africa and Global Apartheid: Continental and International Policies and Politics, Uppsala, Nordiska Afrikainstitutet.

(2013), «Debt, Uneven Development and Capitalist Crisis in South Africa: From Moody's Macroeconomic Monitoring to Marikana's Microfinance Mashonisas», Third World Quarterly, vol. 34, núm. 4.

Bond, Patrick, Trevor Ngwane y Baruti Amisi (2010), Xenophobia and Civil Society: Why did it Happen?, Durban, University of KwaZulu-Natal Centre for Civil Society, en file://C:/Users/carsc/Documents/Elibrary/Authors/Bond/Xenophobia \%205_Why_did_it_happen.pdf

Bourdieu, Pierre (1999), Acts of Resistance: Against the Tyranny of the Market, Nueva York, The New Press.

Bramble, Tom (2003), «Social Movement Unionism Since the Fall of Apartheid: The Case of NUMSA on the East Rand», en Tom Bramble y Franco Barchiesi (eds.), Rethinking the Labour Movement in the "New South Africa", Londres, Ashgate.

BRECHT, Berthold (1967), Gesammelte Werke in acht Bänden, Frankfurt, Suhrkamp.

Buhlungu, Sakhela (2010), A Paradox of Victory: COSATU and the Democratic Transformation in South Africa, Scotsville, University of KwaZulu-Natal Press.

Chatterjee, Partha (2002), The Politics of the Governed, Nueva York, Columbia University Press.

Conen, Robert (2013), «The Marikana Tragedy: South Africa's Social Contract with its Working Poor Breaks Down", Inroads, núm. 32.

CoHen, Robin (1987), The new helots: migrants in the international division of labour, Gower Publishing, Londres.

Cottle, Eddie y Mauricio Rombaldi (2014), «Lessons from South Africa's FIfA World Cup. Brazil and its Legacy for Labour», Wits, The Global Labour University, en http://www.global-labour-university.org/fileadmin/Summer_School_2014/ EddieCottleCETIMbook2013FINAL.pdf

CrusH, Jonathan (1999), «Fortress South Africa and the Deconstruction of Apartheid's Migration Regime», Geoforum, vol. 30, núm. 1. 
(2000), "The Dark Side of Democracy: Migration, Xenophobia and Human Rights in South Africa», en Reginald Appleyard (ed.), The Human Rights of Migrants, Offprint of International Migration, vol. 38, núm. 6, Special Issue 3.

(2003), «South Africa: New Nation, New Migration Policy», Migration Information Source, en http://www.migrationinformation.org/profiles/

Crush, Jonathan y Pendleton Wade (2004), «Regionalizing Xenophobia? Citizen Attitudes to Immigration and Refugee Policy in Southern Africa», Creda Communications, en http://www.queensu.ca/samp/sampresources/samppublications/policyseries/Acrobat30.pdf

Desal, Ashwin (2002), We Are the Poors. Community Struggles in Post-Apartheid South Africa, Nueva York, Monthly Review Press.

Desal, A. y S. Walsh (2010), «Knowledge \& Power in South Africa: Xenophobia and Survival in the Post-Apartheid State (quotes from on line version at academia. edu)», en A. Choudry y D. Kapoor (eds.), Global Perspectives on Social Movements and Knowledge Production: Learning from the Ground Up, Nueva York, Palgrave MacMillan.

Dollery, Brian (2006), «A History of Inequality in South Africa, 1652-2002. Review Note», South African Journal of Economics, vol. 71, núm. 3.

Dube, Phephelaphi (2014), "The 'Undesirable' Immigration Regulations 2014», Cape Town, Centre for Constitutional Rights, en http://www.cfcr.org.za/index. php/latest/293-article-the-undesirable-immigration-regulations-2014

Evans, Jessica (2010), "The Neoliberal Turn in the SADC. Regional Integration and Disintegration", 1 (Fall 2010), pp. 101-129, en http://carleton.ca/africanstudies/wp-content/uploads/5-Evans-Jessica-2010-The-neoliberal-turn-in-theSADC-Regional-integration-and-disintegration-Nokoko-1.pdf

Fanon, Frantz (1967), Black Skin, White Masks, Nueva York, Growe.

Forrest, Kally (2013), «Marikana was not Just about Migrant Labour», Mail \& Guardian, en http://mg.co.za/article/2013-09-13-00-marikana-was-not-just-aboutmigrant-labour

Frankel, Philip (1979), "The Politics of Passes: Control and Change in South Africa», The Journal of Modern African Studies, vol. 17, núm. 2.

(2013), Between the Rainbows and the Rain. Marikana, Migration, Mining, and the Crisis of Modern South Africa, Johannesburgo, Agency for Social Reconstruction.

FudGe, Judy y Kendra Strauss (2013), «Temporary Work, Agencies and Unfree Labour. Insecurity in the New World of Work», en Judy Fudge y Kendra Strauss

$40 \frac{\text { PRIMER SEMESTRE } 2015}{\text { MIGRACIÓN Y DESARROLLO NÚM. } 24}$ 
(eds.), Temporary Work, Agencies, and Unfree Labor. Insecurity in the New World of Work, Nueva York, Routledge.

Galabuzi, Grace-Edward (2006), Canada's Economic Apartheid: The Social Exclusion of Racialized Groups in the New Century, Canadian Scholars», Toronto, Press Inc. Gelb, Steve (1987), «Making Sense of the Crisis», Transformation, vol. 12, núm. 5. Gentle, Lenny (2011), "Poverty and Social Movements», en Brij Maharaj, Desai Ashwin y Patrick Bond (eds.), Zuma's Own Goal. Losing South Africa's 'War on Poverty', Asmara, Africa World Press.

Gilroy, Paul (1987), There Ain't No Black in the Union Jack, Londres, Hutchinson.

Gordon, Steven (2010), «Migrants in a State of Exception», Transcience Journal, vol. 1, núm. 1.

Gramsci, Antonio (1971), «Selections from the Prison Notebooks of Antonio Gramsci», en Hoare Quinton y Geoffrey Nowell Smith (eds.), International Publishers, Nueva York.

Greenfield, Daniel (2014), Frontpage, 24 de febrero, en http://www.frontpagemag. com/2014/dgreenfield/saudi-arabia-the-middle-easts-real-apartheid-state/

Hart, Gillian (2013), Rethinking the South African Crisis: Nationalism, Populism, Hegemony, Geographies Justice and Social Transformation 20, Georgia, University of Georgia Press.

Harvey, David (2005), A Brief History of Neoliberalism, Oxford, Oxford University Press.

Henrard, Kristin (1995-96), "The Internally Displaced in South Africa (1) The Strategy of Forced Removals and Apartheid», Jura Falconis, vol. 32, núm. 4.

Hietalahti, Johanna (2013), "Trade-Offs, Rights and Responsibilities in the Business of Microcredit. A Case Study from South Africa. Academic Dissertation», Helsinki, Department of Political and Economic Studies, en https://helda.helsinki.fi/ bitstream/handle/10138/38909/Hietalahti_dissertation.pdf? sequence $=1$

Hlatshwayo, Mondli (2010), "Cosatu's Attitudes and Policies Towards External Migrants», en Sakhela Byhlungu y Tshoaedi Malehoko (eds.), cosatu's Contested Legacy: South African Trade Unions in the Second Decade of Democracy, Ciudad del Cabo, HSRc Press.

Holmes, Douglas R. (2000), Integral Europe: Fast-Capitalism, Multiculturalism, Neofascism, Princeton y Oxford, Princeton University Press.

Hunt, Jemini (2007), «Brazil is not Just Samba, Sun and Indulgence. The Poor Remain in Slavery», The Guardian, 26 de agosto, en http://www.theguardian.com/ world/2007/aug/26/brazil.theobserver 
KASRILS, Ronnie (2013), "How the ANC's Faustian Pact Sold out South Africa's Poorest», The Guardian, 24 de junio, en http://www.theguardian.com/commentisfree/2013/jun/24/anc-faustian-pact-mandela-fatal-error

KotA, Ayanda (2014), «Some Lessons for South Africa's Sectarian Middle-Class Lefties», Ground Up, en http://groundup.org.za/article/some-lessons-south-africas-sectarian-middle-class-lefties_2506

LAClau, Ernesto (1977), Politics and Ideology in Marxist Theory: Capitalism, Fascism, Populism, Londres, NLB.

Landau, Loren B. (2011), Exorcising the Demons within. Xenophobia, Violence and Statecraft in Contemporary South Africa, Johannesburgo, Wits University Press.

Legassick, Martin (1974), «South Africa: Capital Accumulation and Violence», Economy and Society, vol. 3, núm. 3.

LeGASSICK, Martin y Wolpe Harold (1976), «The Bantustans and Capital Accumulation in South Africa», Review of African Political Economy, núm. 7.

Lipietz, Alain (1982), «1. Towards Global Fordism 2. Marx or Rostov?», New Left Review, núm. 132.

Maharaj, Brij, Desai Ashwin y Patrick Bond (eds.) (2011), Zuma's Own Goal. Losing South Africa's "War on Poverty», Asmara, Africa World Press.

Maharajh, Rasigan (2011), Innovating Beyond Racial Capitalism: A Contribution Towards the Analysis of the Political Economy of Post-Apartheid South Africa, Lund, Lund University Press.

Mamdani, Mahmood (1996), Citizen and Subject: Contemporary Africa and the Legacy of Late Colonialism, Princeton, Princeton University Press.

Mandela, Nelson (2012), «Ubuntu Told by Nelson Mandela», disponible en https:// www.youtube.com/watch? $\mathrm{v}=\mathrm{HED} 4 \mathrm{~h} 00 \mathrm{xPPA}$

Mapokgole, Reshoketswe B. (2014), "There is No Black in The Rainbow (Nation): A Bikoist and Fanonian Approach To Understanding 'Xenophobic' Violence in South Africa", Londres y Ciudad del Cabo, Trinity College, en http://digitalrepository.trincoll.edu $/ \mathrm{cgi} / \mathrm{viewcontent}$.cgi .article $=1437 \&$ context $=$ theses

MaraIs, Hein (2001 [1998]), South Africa. Limits to Change: The Political Economy of Transition. Revised and Expanded New Edition, Ciudad del Cabo, University of Cape Town Press.

Marx, Karl (1976), Capital. A critique of political economy, Penguin books limited, Londres.

$42 \frac{\text { PRIMER SEMESTRE } 2015}{\text { MIGRACIÓN Y DESARROLLO NÚM. } 24}$ 
Massey, D. y N. Denton (1993), American Apartheid: Segregation and the Making of the Underclass, Cambridge, Harvard University Press.

Matsinhe, David M. (2011), Apartheid Vertigo. The Rise in Discrimination Against Africans in South Africa, Interdisciplinary Research Series in Ethnic, Gender and Class Relatinos, Farnham, Ashgate.

MBAthA, Ndumiso A. (2013), "Chronicles of a Slave Chain», Toughts From the Bishop, en https://ndumisombatha.wordpress.com/2013/09/24/chronicles-of-afree-slave/

Mbeki, Moeletsi (2009), Architects of Poverty. Why African Capitalism Needs Changing, Johannesburgo, Picador África.

Mвемвe, Achille (2015), "Achille Mbembe Writes about Xenophobic South Africa», Africa is a Country, en http://africasacountry.com/achille-mbembe-writesabout-xenophobic-south-africa/

Miles, Robert (1987), Capitalism and unfree labour; anomaly or necessity?, Tavistock, Nueva York.

MingXitama, Andile (2008), "We are not all Like that. Race, Class and Nation after Apartheid», en Shireen Hassim, Kupe Tawana y Erik Worby (eds.), Go Home or Die Here, Johannesburgo, Wits University Press.

Miraftab, Faranak (2004a), "Neoliberalism and Casualization of Public Sector Services: The Case of Waste Collection Services in Cape Town, South Africa», International Journal of Urban and Regional Research, vol. 28, núm. 2.

(2004b), «Invited and Invented Spaces of Participation: Neoliberal Citizenship and Feminists», Expanded Notion of Politics», Wagadu, núm. 1.

(2009), «Insurgent Planning: Situating Radical Planning in the Global South», Planning Theory, vol. 32, núm. 8.

Misago, Jean Pierre, Loren B. Landau y Tamlyn Monson (2009), Towards Tolerance, Law, and Dignity: Addresssing Violence Against Foreign Nationals in South Africa, Johannesburgo, IOM and the Department for International Development (DFID), University of the Witwatersrand.

Miworc (2014), «Migrancy and Labour in the Hospitality Sector in South Africa», African Centre for Migration and Society (ACMS), en http://www.miworc.org.za/?.e=30

Munakamwe, Janet y Jinnah Zaheera (2014 [en preparación]), A Bitter Harvest: Migrant Workers in the Commercial Agricultural Sector in South Africa, African Centre for Migration and Society (ACMS), Johannesburgo, en http://www.agbiz.co.za/ uploads/documents/news/Newsletter/2014/November/14_11_06\%20report\%20-\%20foreign\%20labour\%20in\%20the\%20agriculture\%20sector.pdf 
Munusamy, Ranjeni (2015), «Xenophobia and Populism: The anc, efF and United Front's Complex Chase for SA's Mass Base», Daily Maverick, 28 de mayo, en http:// www.dailymaverick.co.za/article/2015-01-30-xenophobia-and-populism-theanc-eff-and-united-fronts-complex-chase-for-sas-mass-base/\#.VWd7ukb-lc8

Narsiah, Sagie (2002), «Neoliberalism and Privatisation in South Africa», GeoJournal, vol. 57 , núm. 1-2.

NAUDÉ, Wim (2013), «Governance Failure: Decline of Innovation and Entrepreneurship in South Africa», United Nations University, en http://unu.edu/publications/articles/governance-failure-in-south-africa.html

Ndletyana, Mcebisi (2011), "Is SA Becoming a Sub-imperial Hegemon?», The Sunday Independent, 5 de septiembre, en http://www.iol.co.za/sundayindependent/issa-becoming-a-sub-imperial-hegemon-1.1131327\#.VRpr4eH-lpt

Neocosmos, Michael (2006), From «Foreign Natives'to Native Foreigners»: Explaining Xenophobia in Post-Apartheid South Africa, Dakar, Codesria.

(2008), «Neocosmos: The Politics of Fear and the Fear of Politics (Essay on the Pogroms)", Abahlali baseMjondolo. Home of the Abahlali baseMjondolo Shackdwellers' Movement South Africa, en http://abahlali.org/node/3616/

(2011), "Transition, Human Rights and Violence: Rethinking a Liberal Political Relationship in the African Neo-colony», Interface: a Journal for and About Social Movements, vol. 3, núm. 2.

(2015), "The Sickness of Xenophobia, and the Need for a Politics of Healing», The Daily Maverick, en http://www.dailymaverick.co.za/opinionista/201502-02-the-sickness-of-xenophobia-and-the-need-for-a-politics-of-healing/\# . VQURZeGBtc8

NGwane, Trevor (2011), «Ideologies, Strategies and Tactics of Township Protest», en Brij Maharaj, Desai Ashwin y Patrick Bond (eds.), Zuma's Own Goal. Loosing South Africa's War on Poverty, Asmara African, World Press.

O'Brien, Martin y Sue Penna (1998), Theorising Welfare. Enlightenment and Modern Society, Londres, Sage.

Paret, Marcel (2013), "Precarious Labor Politics: Unions and the Struggles of the Insecure Working Class in the USA and South Africa», Critical Sociology, printed online, 17 de abril, en http://crs.sagepub.com/content/early/2013/04/17/ 0896920513483149.abstract

Peberdy, Sally (2001), «Imagining Immigration: Inclusive Identities and Exclusive Policies in Post-1994 South Africa», Africa Today, vol. 48, núm. 3.

$44 \frac{\text { PRIMER SEMESTRE } 2015}{\text { MIGRACIÓN Y DESARROLLO NÚM. } 24}$ 
PhilLIPS, Nicola (2013), «Unfree Labour and Adverse Incorporation in the Global Economy: Comparative Perspectives on Brazil and India», Economy and Society, vol. 42, núm. 2.

Polanyi, Karl (1957 [1944]), The Great Transformation: The Political and Economic Origins of Our Time, Boston, Beacon Press.

RansLem, Duncan (2015), «Temporary 'Relocation: Spaces of Contradiction in South African law'», International Journal of Law in the Built Environment, vol. 7, núm. 1.

Richmond, A. H. (1994), Global Apartheid: Refugees, Racism, and the New World Order, Toronto, Nueva York y Oxford, Oxford University Press.

SATGar, Wishwas (2011), "Vishwas Satgar: Reclaiming a Vision of Hope and a Life of Dignity: Neoliberal South Africa and the Narrowing of Democratic Space», Conferencia magistral realizada en la Conference of the Democratic Left, Constitutionally Speaking, en http://constitutionallyspeaking.co.za/vishwas-satgarreclaiming-a-vision-of-hope-and-a-life-of-dignity-neoliberal-south-africa-andthe-narrowing-of-democratic-space/

(2012), «Beyond Marikana: The Post-Apartheid South African State», Africa Spectrum, vol. 47, núm. 2-3.

SAUL, John S. y Patrick Bond (2014), South Africa-The Present as History. From Mrs. Pless to Mandela \& Marikana, Nueva York, Rochester.

SCHIERUp, Carl-Ulrik (2015), «A Toxic Embrace: Migration, Labour, and the Rainbow Nation's Neoliberal Pact», en Carl-Ulrik Schierup et al. (eds.), Migration, Precarity, and Global Governance: Challenges for Labour, Oxford, Oxford University Press.

SegATII, Aurelia (2011), "Reforming South African Immigration Policy in the Postapartheid Period (1990-2010)», en Aurelia Segatti y Loren B. Landau (eds.), Contemporary Migration to South Africa: A Regional Development Issue, Washington, International Bank for Reconstruction and Development, The World Bank.

Sewell, Rob (2004), «Lenin on the National Question», Defence of Marxism, en http:// www.marxist.com/lenin-national-question160604.htm

SivanAndan, Ambalavaner (2001), "Poverty is the New Black», Race \& Class, vol. 43, núm. 1.

Stovo, Joe (1988), "The South African Working Class and the National Democratic Revolution", African Socialism Archive, en https://www.marxists.org/subject/ africa/slovo/1988/national-democratic-revolution.htm

Standing, Guy (2011), The Precariat. The New Dangerous Class, Londres, Bloomsbury Academic. 
TATI, Gabriel (2008), «The Immigration Issues in the Post-Apartheid South Africa: Discourses, Policies and Social Repercussions", Géopolitique et Populations, núm. 3. Terreblance, Sampie (2003), A History of Inequality in South Africa 1652-2002, Durban, University of KwaZulu-Natal Press.

Theron, Jan (2010a), «Sour Grapes», Law, Democracy and Development, núm. 14. (2010b), «Informalization from Above, Informalization from Below: The Options for Organization", African Studies Quarterly, vol. 11, núm. 2-3.

Tobias, Saul (2012), «Neoliberal Globalization and the Politics of Migration in SubSaharan Africa», Journal of International and Global Studies, 4(1): 1-16.

Trimikuiniotis, Nicos, S. Gordon y B. Zondo (2008), «Globalisation and Migrant Labor in a Rainbow Nation: a Fortress South Africa?», Third World Quarterly, vol. 29 , núm. 7.

Tshabalala, Xolani (2015), «Waiting on the Move: The Social Life of Mobility Governance Across the Beitbridge Border», REMESO, Linköping University.

Tsianos, Vassilis y Serhat Karakayali (2010), "Transnational Migration and the Emergence of the European Border Regime: An Ethnographic Analysis», European Journal of Social Theory, vol. 13, núm. 3.

Tutu, Desmond (1996), The Rainbow People of God, Colorado, Springs Image. (1999), No Future without Forgiveness, Nueva York, Doubleday.

UK, Communist Party (2014), The New Scramble for Africa, Londres, International Commission, Communist Party of Britain.

UPM (2013), «Unemployed People's Movement», en http://makana.wikifoundry. $\mathrm{com} /$ page/Unemployed + People\%27s + Movement

Wentzel, Marie y Tlabela Kholadi (2006), «Historical Background to South African Migration", en Pieter Kok et al. (eds.), Migration in South and Southern Africa. Dynamics and Determinants, Ciudad del Cabo, HSRC Press.

Willén, Julia (2015), "The Curse of the Nation State and History as Remedy? Xenophobia and Migritude in Post-Apartheid South Africa», Discover Society, núm. 17.

Wolpe, Harold (1972), "Capitalism and Cheap Labour-Power in South Africa: From Segregation to Apartheid», Economy and Society, vol. 1, núm. 4.

Xulu, Nomkhosi (2010) «COSATU and Internal Migrant Workers: Old Fault Lines, New Dilemmas», en Sakhela Bulungu y Malehoko Tshoaedi (eds.), Cosatu's Contested Legacy: South African Trade Unions in the Second Decade of Democracy, Ciudad del Cabo, HSRC Press. 